\title{
A COMPARISON OF THE PUSH AND PULL PRODUCTION SYSTEMS AT THEIR OPTIMAL DESIGNS UNDER THE ECONOMIC CONSIDERATION
}

\author{
N. Chiadamrong* and P. Kohly \\ Industrial Engineering Program, Sirindhorn International Institute of Technology \\ Thammasat University, Pathumthani, 12121, Thailand
}

Received 01 June 2005

\begin{abstract}
The term "push" and "pull" have been used to explain a wide variety of production inventory systems. The distinction refers to a specific attribute, which can be identified by observing the mechanisms for controlling material flow on the shop floor and a specific policy for the management of inventories and production schedules. This paper gives an attempt to compare these systems under their optimal settings under a constraint resource. Two optimal-seeking methods (Taguchi method and Response Surface Methodology) are used to suggest the optimized design of the system under an economic term, which is the profit generated from the system. Then, a fair comparison can be made where each system is operating at its optimal design. Results from this study will reveal an interesting outcome, letting us know the impact of the push and pull mechanisms on the systems' operating costs as well as their profits.
\end{abstract}

\section{INTRODUCTION AND BACKGROUND OF THE PROBLEM}

Several papers in the past have focused on comparing push and pull systems. Sarker and Fitzsimmons [1] used simulation to measure the performance of the push and pull systems under different coefficients of variation of the processing times. The results show that a pull system is always better at minimum work-in- process, but on the other hand it is less efficient than the push system, especially at higher coefficients of variation. Lee [2] examined the performance pf the push and pull systems under different load (demand) conditions. Effectiveness measures monitored include job throughput, process utilization and inventory levels.

A production system under the investigation is known as a flow line when all stages are arranged in series and all products manufactured in the system follow the same sequence of processes. A flow line is usually designed to be dedicated to a particular product. The maximum output of the flow line is influenced by the slowest operation in the line and hence considerable efforts are usually made to balance the line and reduce the affects of the bottleneck on the line.

\footnotetext{
* Corresponding author e-mail: navee@siit.tu.ac.th
} 
This bottleneck is defined as a point in the manufacturing process that holds down the amount of products that a factory can produce [3]. The great majority of previous studies of production lines have assumed that real production lines are either perfectly balanced or are nearly so. This claim is not based on empirical evidence, but on the assumption that unbalanced lines do not exist because they are less efficient than balanced lines. Even though, the bottleneck is undesirable, it is difficult to avoid, especially under the flow line where all products need to follow the same sequence of different processes. Alleviation of such problems requires not only explicit understanding of the entire process, but also a powerful production control system.

Because of the large number of parameters involved and the complexity of their relationships, it is found that the performance of each system is varied according to their parameter settings. In addition, one system may be better at one performance measure but worse at another performance measure. As a result, it would be unfair to compare these systems at just one performance measure and conclude that it is better. To be fair, both systems should be compared under the same basis at their best parameter settings and the judging criteria should look at overall criteria (i.e., economic consideration in terms of profit) rather than only one performance measure based on just one criteria.

\section{MODEL CHARACTERISTICS}

This study focuses on the unbalanced line or a line in which one station has its mean processing time longer than all other stations. The decision to be made is to determine the parameter settings that yield maximum profit for the push driven flow line and similarly for the pull driven flow line. Owing to the complexity of this system, simulation is employed as a tool for analysis. All experimental models are developed using SIMAN simulation language [4]. All simulation runs we made were for 10 replications with the replication length of 115,200 minutes (1 year) in which a $95 \%$ confidence interval for the flow times, based on 10 replications with different seeds, has a width less than 0.05 . Fig. 1 shows the layout of the flow line under the push production control system and Fig. 2 shows the layout of the flow line under the pull control system.

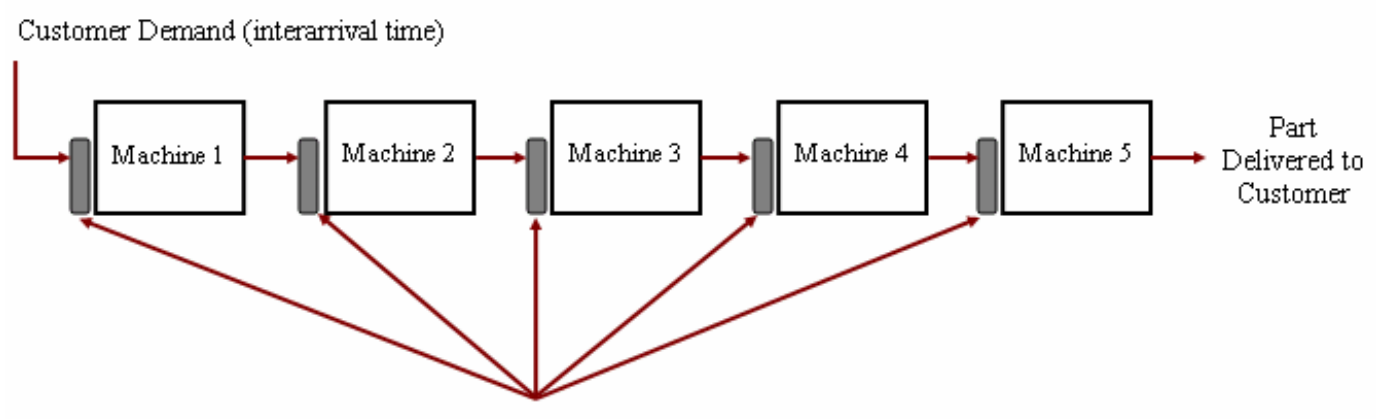

Equal Buffer Space in front of each machine

Fig. 1: $\quad$ Layout of push driven flow line 
Part request is matched with kanban and part is sent to queue in front of the machine and request is sent to the prior machine

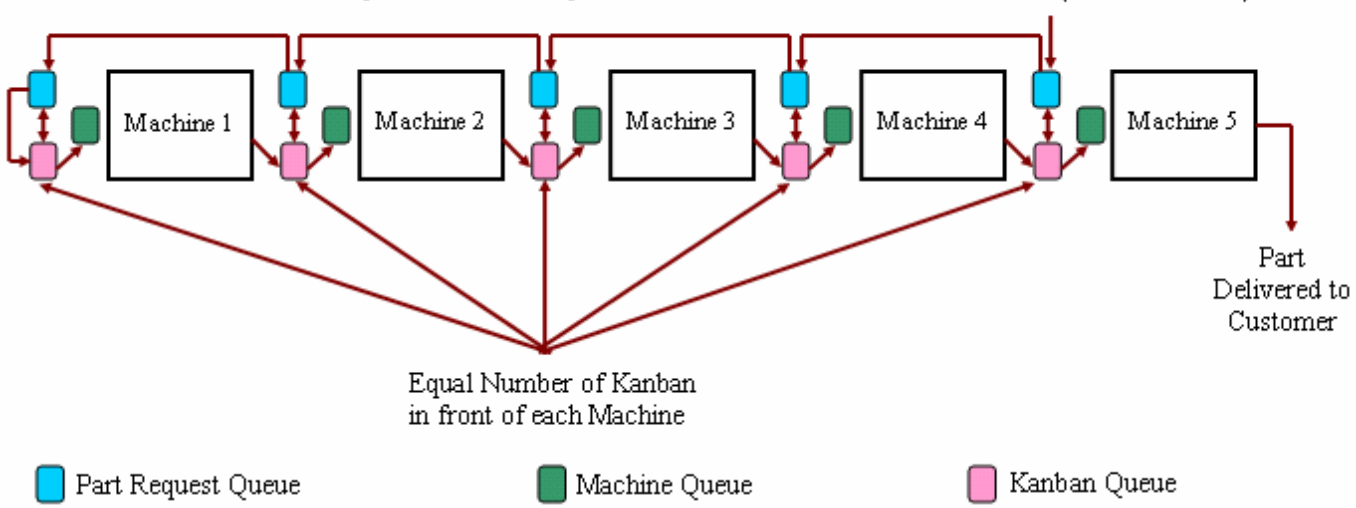

Fig. 2: $\quad$ Layout of pull driven flow line

The procedure of the push system is relatively simple. Each order on entry (one unit of part) into the system is queued at the first required process. If the number of parts waiting to be processed in this queue reaches the maximum buffer size, these new arrival parts cannot join the queue and they are considered as lost sales. In an asynchronous line, each machine can pass parts on when its processing is completed, as long as a buffer space is available (or, when no buffer exists, the downstream machine is idle). This type of line is subject to manufacturing blocking and starving [5]. Too small buffer space at one station may cause the preceding station to stop (blocking) when the upstream station is unable to transfer parts to the blocking station. In contrary, too large buffer space would not be economical to operate. On completion of a process, the part proceeds to subsequent processes one at a time until it exists from the line. Due-date of each job is calculated using the total work content method with the multiplier of 25. In Blackstone et al. [6], it is pointed out that this is the most rational method of assigning internally determined duedates. As a result, when parts finish beyond their due-dates, the penalty cost would be charged.

For the pull system, activities at the process stations are triggered by depleted kanban stock at the process station. Inventory level between stages is controlled by the number of kanbans initially allocated. A kanban is sent from a machine to the preceding machine to initiate production of a unit or a specified number of units. In an ideal pull system, one unit of inventory at each production stage is enough; but, this goal is not achievable in real manufacturing environments due to variation in demand and processing times. Thus, when the demand and processing time are stochastic, the determination of the number of kanbans that will optimize system performance is an issue of considerable interest for practitioners and researchers alike. Similarly, lost sales and penalty cost would occur if orders are over-flowed from the system and parts are finished beyond their due-dates. 
The analysis of this study starts with 5 station flow line with a single bottleneck station where one of the machines (either machine 1, machine 3 or machine 5) is assigned to be a bottleneck station. However, this result should also be able to generalize to other longer line cases. Powell and Pyke [7] indicated that the general behavior of unbalanced lines is not so much insensitive to line length as other bottleneck factors especially the severity and position can have far more influence on the output of the line than the effect of the line length. As a result, the position and its severity of the bottleneck station are considered to be one of the controllable factors for designing its best setting where its negative effect is minimum. As a result, the comparison between push and pull systems in this study may not compare both systems at the same location of the bottleneck but at its optimal location.

In a normal circumstance, the bottleneck station's processing time is twice longer than the ones from other workstations (mean processing time of 10 minutes at the bottleneck station as compared to 5 minutes at other stations). To be fair, an attempt to reduce this severity must incur some expenses otherwise the optimal setting of this factor would always suggest no bottleneck case. As a result, bottleneck processing time reduction cost of 18,000 Baht is assumed to pay for every 0.1 minute of bottleneck time reduction. The machine operation times are lognormally distributed with a standard deviation that is $20 \%$ of the processing time. This is because its positively skewed has only positive processing time and its ability to model high variability stations. Buzacott and Shantikumar [8] suggest the real workstation time exhibit positive skewness as does the lognormal distribution. However, the inter-part arrival time, mean time between failure and mean time to repair follow exponential distribution.

\section{ORTHOGONAL INNER AND OUTER ARRAY}

As, the controllable factors include part inter-arrival time, buffer size (for push system) or number of kanbans (for pull system), the position of the bottleneck station in the flow line and the severity of the bottleneck (bottleneck processing time), mean time between failure (MTBF) and mean time to repair (MTTR) are treated as uncontrollable factors (noise). Table 1 and 2 show the associated levels for each factor. Each of the controllable factors is to be tested at three levels and the noise factors are varied over two levels. The idea is to obtain a robust design that will be insensitive to the noise factors during the actual operation. Due to four noise combinations and 81 controllable factorial combinations, 342 experimental conditions result for this experiment.

Table 1: Controlled factors and their assigned levels

\begin{tabular}{lccc}
\hline \multirow{2}{*}{ Controlled factors } & Low & Medium & High \\
\cline { 2 - 4 } & 5 minutes & 10 minutes & 15 minutes \\
\hline Part inter-arrival time & 5 & 10 & 15 \\
$\begin{array}{l}\text { Buffer size (for push system) or } \\
\text { number of kanbans (for pull system) }\end{array}$ & Machine 1 & Machine 3 & Machine 5 \\
Position of the bottleneck & 5 minutes & 7.5 minutes & 10 minutes \\
Bottleneck processing time & & & \\
\hline
\end{tabular}


Table 2: $\quad$ Uncontrolled factors and their assigned levels

\begin{tabular}{lcc}
\hline \multirow{2}{*}{ Uncontrolled factors } & Low & Levels \\
\cline { 2 - 3 } & 500 minutes & 800 minutes \\
\hline Mean time between failure (MTBF) & 30 minutes & 60 minutes \\
\hline Mean time to repair (MTTR) & & \\
\hline
\end{tabular}

\section{PROFIT MODEL}

The profit model is constructed and used to convert the performance of each design into the monetary term. Table 3 presents the cost structure used in the experiment.

Profit $=$ Revenue - Total costs

Revenue $=F \times P$

where $\quad F=$ the number of finished units

$P=$ the selling price per unit (Baht)

Total Costs $=\sum_{i=1}^{m} O_{i}+R m+\sum_{i=1}^{m} R e_{i}+\sum_{i=1}^{m} H_{i}+\sum_{i=1}^{m} I_{i}+L s+L p+P r$

$O_{i}=O T_{i} \times O_{c}$

where $\quad O_{i}=$ Total operating cost of machine $i$ (Baht)

$O T_{i}=$ Total operating time of machine $i$ (minutes)

$O_{c}=$ Machine utility cost per minute (Baht)

$R m=F \times R c$

where $\quad R m=$ Total raw material cost (Baht)

$F=$ Number of finished units

$R c=$ Raw material cost per unit (Baht/unit)

$R e_{i}=R T_{i} \times R P_{c}$

where $R e_{i}=$ Total repairing cost of machine $i$ (Baht)

$R T_{i}=$ Total repair time of machine $i$ (minutes)

$R P_{c}=$ Machine repair cost per minute $($ Baht $/ \mathrm{min})$

$H_{i}=Q T_{i} \times U c \times C_{T p}$

where $\quad H_{i}=$ Holding cost of parts waiting in a queue in front of machine $i$ (Baht)

$Q T_{i}=$ Total process waiting time of parts waiting in a queue in front of machine $i$ (minutes)

$U c=$ Part unit cost (Baht)

$C_{T p}=$ Cost of capital due to part holding (\%)

$I_{i}=\left\{\left[\left(\left(1-U_{i}\right) \times t\right)+I T_{i}\right] \times E_{m} \times\left(D \times M_{c} \times m\right)\right\} \times C_{T i}$

where $\quad I_{i}=$ Total idle cost for machine $i$ (Baht)

$U_{i}=$ Utilization of machine $i(\%)$ 
$t=$ Replication length (minutes)

$I T_{i}=$ Total blocking time of machine $i$ (minutes)

$E_{m}=$ Machine efficiency (assumed to be equal for all machines)

$D=$ Depreciation rate

$M_{c}=$ Machine investment cost (assumed to be equal for all machines in Baht)

$m=$ Total number of machines

$C_{T i}=$ Cost of capital due to machine idleness (\%)

$L s=O P \times L S_{c}$

where $\quad L s=$ Total lost sales cost (Baht)

$O P=$ Number of overflow orders from the system (units that cannot enter the line)

$L S_{c}=$ Lost sales cost per unit (Baht/unit)

$L p=L T \times L P_{c}$

where $\quad L p=$ Total late penalty cost (Baht)

$L T=$ Total late time (minutes)

$L P_{c}=$ Late penalty cost per minute (Baht/minute)

$\operatorname{Pr}=10 \times(10-P t) \times B r$

where $\quad P r=$ Total bottleneck processing time reduction cost (Baht)

$P t=$ Processing time of the bottleneck (minutes)

$\mathrm{Br}=$ Processing time reduction cost (Baht per 0.1 minute reduction time)

Table 3: Cost structure

\begin{tabular}{ll}
\hline Selling price per unit $(P)$ & $400 \mathrm{Baht} /$ unit \\
Raw material cost per unit $(R c)$ & $50 \mathrm{Baht} / \mathrm{unit}$ \\
Machine utility cost per hour $(O c)$ & $40 \mathrm{Baht} / \mathrm{hour}$ \\
Part unit cost $(U c)$ & $200 \mathrm{Baht} / \mathrm{unit}$ \\
Machine efficiency $\left(E_{m}\right)$ & $90 \%$ \\
Depreciation $(D)$ & $20 \%$ per year \\
Cost of Capital due to holding time $\left(C_{T_{p}}\right)$ & $480 \%$ per year \\
Cost of Capital due to machine idleness $\left(C_{T i}\right)$ & $20 \%$ per year \\
Machine investment cost $(M c)$ & $1,000,000 \mathrm{Baht}$ \\
Total number of machines $(m)$ & $5 \mathrm{machines}$ \\
Repair cost per minute $\left(R P_{c}\right)$ & $2.5 \mathrm{Baht} / \mathrm{minute}$ \\
Processing time reduction cost $(B r)$ & $18,000 \mathrm{Baht} / 0.1$ minute \\
Lost sales cost $\left(L S_{c}\right)$ & $50 \mathrm{Baht} / \mathrm{unit}$ \\
Late penalty cost $\left(L P_{c}\right)$ & $2 \mathrm{Baht} / \mathrm{minute}$ \\
\hline Remark: $\mathbf{1} \mathbf{U S} \$ \mathbf{4 0}$ Baht & \\
\hline
\end{tabular}




\section{OPTIMIZATION OF THE SYSTEM PARAMETER SETTINGS USING AN INTEGRATED APPROACH}

An integration of Taguchi method and Response Surface Methodology (RSM) is used to determine the optimal combination of system parameters. Profit received from the system will be used as the overall performance indicator when comparing a push driven flow line with a pull driven flow line. Many successful application of Taguchi method have been reported over the last fifteen years [9]. However, when the input factors are quantitative and continuous, the RSM is better suited. RSM studies the local geography of the response surface near the optimal value through the response function. It is also useful for modeling and analyzing applications where a response of interest is influenced by several variables [10].

Due to the nature of our problem where both qualitative and quantitative factors are present simultaneously, Taguchi method and RSM can be used to supplement each other to give the best solution. The Taguchi method can be used to optimize qualitative variables (i.e., the location of the bottleneck station) while RSM fine-tunes the quantitative results derived from the Taguchi method and strives for better solution. Shang [11] and Shang and Tadikamalla [12] have employed this approach by combining the Taguchi and RSM to study the multi-criteria performances of manufacturing systems. Their studies have proven that the combined Taguchi and RSM technique can offer a practical method where both qualitative and quantitative factors are concerned and combining both methods helps us achieve their fullest potential. Next, this integrated approach will be introduced to determine the optimal system parameters for maximizing the profit for both push and pull systems.

\section{PUSH SYSTEM}

\subsection{Taguchi method for experimental design}

The primary aim of the Taguchi method is to minimize variations in the output when the noise is presented in the process. A signal-to-noise $(S / N)$ ratio is used to find the most robust combination. $S / N$ is calculated depending on the objective of the problem. In this case, the profit has the bigger-the better characteristic. Hence, the following equation is used.

$$
S / N_{L T B_{i}}=-10 \log \frac{\sum_{j=1}^{n}\left(1 / y_{i j}{ }^{2}\right)}{n}
$$

where:

$S / N_{L T B_{i}}$ is signal-to-noise ratio for larger-the-better case;

$y_{i j}$ is the response (profit) from the $i^{\text {th }}$ combination of control factors and $j^{\text {th }}$ combination of noise factors;

$n$ is the total number of combinations of noise factors for each combination of control factors.

In order to find the best parameter setting using Taguchi method, it is necessary to create plots of the $S / N$ ratios of each controllable factor. The optimal set points of the controlled factor levels are the ones at which the $S / N$ ratio is maximized. The values that have been plotted in Fig. 3 can also be seen in Table 4 , where the highest $S / N$ ratios have been highlighted. 

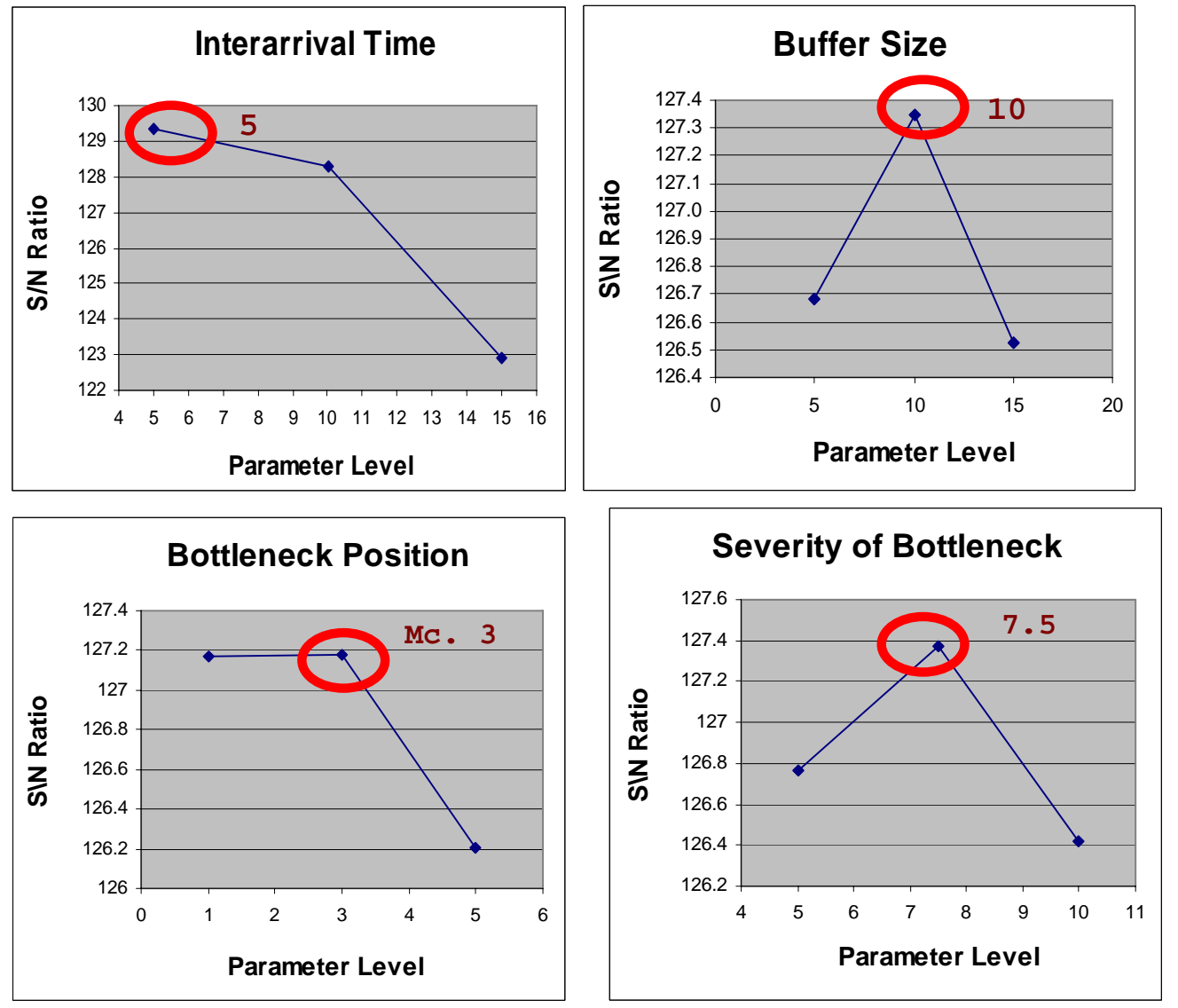

Fig. 3: $\quad$ Taguchi method results for the push system

Table 4: $\quad$ S/N Ratios for all controlled factors of the push system

\begin{tabular}{lccc}
\hline \multirow{2}{*}{ Controllable factors } & \multicolumn{3}{c}{ Level } \\
\cline { 2 - 4 } & Low & Medium & High \\
\hline Inter-arrival time & 129.361 & 128.304 & 122.892 \\
Buffer size & 126.684 & 127.349 & 126.524 \\
Bottleneck position & 127.167 & 127.181 & 126.209 \\
Bottleneck processing time & 126.767 & 127.369 & 126.420 \\
\hline
\end{tabular}

The most robust design as recommended by the Taguchi method is a flow line with inter-arrival time of 5 minutes, buffer size of 10 units, bottleneck located at machine 3 and bottleneck processing time of 7.5 minutes. However, it should be noted that there is no guarantee that choosing these recommended points will lead to maximizing the profit of the line since it may be at a saddle point.

The most robust design as recommended by the Taguchi method is a flow line with inter-arrival time of 5 minutes, buffer size of 10 units, bottleneck located at machine 3 and bottleneck processing time of 7.5 minutes. However, it should be noted that there is no guarantee that choosing these recommended points will lead to maximizing the profit of the line since it may be at a saddle point. 


\subsection{Response Surface Methodology}

Factor levels recommended by the Taguchi method are used in this section as the initial setting. The goal is to further maximize the system's profit if the input factors are controllable and can be varied in a continuous manner. RSM is divided into four phases, where phase 1 is the first order analysis and the second phase is the second order analysis. The third phase finds the optimal solution and the obtained results need to be verified in the fourth phase.

Phase I: First order analysis

\section{Step 1: Range determination}

In this step, the robust design received from Taguchi method is used as the center point. The exploration points are chosen above and below the center point. The region of exploration is set as following: $(2.5,7.5)$ for inter-arrival time (minutes) where the center point is 5 minutes, $(5$, 15) for buffer size where center point is 10 units, bottleneck position fixed at machine 3 and finally $(5,10)$ minutes for bottleneck processing time where the center point is 7.5 minutes.

\section{Step 2: Coding independent variables}

Variables are coded to an interval of $(-1,1)$ so that calculations during this phase can be simplified. The coding is done using the following equation:

$$
X_{i}=\frac{\left(i^{\text {th }} \text { factor's natural value - center point }\right)}{\text { Half the range of the variable }}
$$

The coded variables are: $X_{1}=($ inter-arrival time -5$) / 2.5 ; X_{2}=($ buffer size -10$) / 5 ; X_{4}=$ (bottleneck processing time -7.5 ) / 2.5 where $X_{1}, X_{2}, X_{4}$ are coded variables of part inter-arrival time, buffer size and bottleneck processing time respectively. The factor of bottleneck position $\left(X_{3}\right)$ has been fixed at machine 3 and thus will not be considered as a variable from now onwards.

\section{Step 3: Data collection}

$2^{k}(k=3)$ full factorial design is used and augmented by four center points. Repeat observations at the center are used to estimate the experimental error and to allow for checking the adequacy of the first-order model. Since each design is simulated and averaged under four noise settings, there are 48 experimental conditions in all.

\section{Step 4: First order model fitting}

The data collected in Step 3 is used and a first order model that best fits the data is found. Here $X_{1}, X_{2}$ and $X_{4}$ are the independent variables (controlled factors) and $y$ is the profit. The regression equation is as follows:

$$
y=\beta_{0}+\sum_{i=1}^{k} \beta_{i} x_{i}
$$

where $X_{i}$ is the controlled factor, $\beta_{i}$ is the regression coefficient and $k$ is the number of controlled factors. By using the least square method, the equation of this best fit line is:

$$
y=3,018,024+335,010.7 X_{1}+172,395.6 X_{2}-905,161 X_{4}
$$




\section{Step 5: First order adequacy test}

It is necessary to make sure that the data obtained are relevant and thus Analysis of Variance (ANOVA) is used to determine the model's significance under a $95 \%$ confidence level. The first-order equation gives F-value of 268.057 (p-value of 0.000), which indicates that the model is adequate.

\section{Step 6: Method of steepest ascent}

The path of steepest ascent is the direction in which the response increases most rapidly. Here first we need to select the independent variable that has the largest regression coefficient in the model. This is $X_{4}$ (bottleneck processing time) with $\beta_{4}$ of 905,161 . The coded step size for other variables can be calculated by the following equation:

$$
\Delta X_{i}=\beta_{i} / \beta_{4} \quad \text { for } \quad i=1,2,4 .
$$

Hence,

$$
\begin{aligned}
\Delta X_{1} & =335,010.7 / 905,161=0.370 \\
\Delta X_{2} & =172,395.6 / 905,161=0.190 \\
\Delta X_{4} & =-905,161 / 905,161=-1 .
\end{aligned}
$$

Next the coded variable $\Delta X_{i}$ is converted to natural variable, $N T_{i}$. This is done by multiplying $\Delta X_{i}$ with the actual step size $\left(S_{i}\right)$. The smallest step size for the inter-arrival time is set at 0.1 minutes, for buffer size is set at 1 unit and for bottleneck processing time is set at 0.1 minute.

Therefore,

$$
\begin{aligned}
& \Delta X_{1} S_{1}=0.1 \text { minute } \\
& \Delta X_{2} S_{2}=1 \text { unit } \quad S_{1}=0.1 / 0.370=0.27 \\
& \Delta X_{4} S_{4}=0.1 \text { minute } \text { So, } S_{2}=1 / 0.190=5.26
\end{aligned}
$$

\begin{tabular}{|c|c|c|c|c|c|c|c|c|c|c|}
\hline & \multirow[b]{2}{*}{ Steps } & \multicolumn{4}{|c|}{ Coded variables } & \multicolumn{4}{|c|}{ Natural variables } & \multirow{2}{*}{$\begin{array}{c}\text { Profit } \\
Y \text { (Baht) }\end{array}$} \\
\hline & & $\begin{array}{c}X_{I} \text { (Inter- } \\
\text { arrival) }\end{array}$ & $\begin{array}{c}X_{2} \\
\text { (Buffer } \\
\text { Size) } \\
\end{array}$ & $\begin{array}{c}X_{3} \\
\text { (position) }\end{array}$ & $\begin{array}{c}X_{4} \\
\text { (Severity) }\end{array}$ & $\begin{array}{c}N T_{l} \\
\text { (minutes) }\end{array}$ & $\begin{array}{l}N T_{2} \\
\text { (units) }\end{array}$ & $\begin{array}{c}N T_{3} \\
\text { (position) }\end{array}$ & $\begin{array}{c}N T_{4} \\
\text { (minutes) }\end{array}$ & \\
\hline & Origin & 0 & 0 & Mc 3 & 0 & 5 & 10 & Mc 3 & 7.5 & $3,305,474.078$ \\
\hline $\begin{array}{c}\text { Step } \\
\text { number }\end{array}$ & $\Delta$ & 0.04 & 0.2 & Fixed & -0.04 & 0.1 & 1 & Fixed & -0.1 & \\
\hline 1 & Origin $+1 \Delta$ & 0.04 & 0.2 & Mc 3 & -0.04 & 5.1 & 11 & Mc 3 & 7.4 & $3,411,054.037$ \\
\hline 2 & Origin $+2 \Delta$ & 0.08 & 0.4 & Mc 3 & -0.08 & 5.2 & 12 & Mc 3 & 7.3 & $3,515,785.714$ \\
\hline 3 & Origin $+3 \Delta$ & 0.12 & 0.6 & Mc 3 & -0.12 & 5.3 & 13 & Mc 3 & 7.2 & $3,591,196.623$ \\
\hline 4 & Origin $+4 \Delta$ & 0.16 & 0.8 & Mc 3 & -0.16 & 5.4 & 14 & Mc 3 & 7.1 & $3,686,762.592$ \\
\hline 5 & Origin $+5 \Delta$ & 0.2 & 1 & Mc 3 & -0.2 & 5.5 & 15 & Mc 3 & 7 & $3,794,866.759$ \\
\hline 6 & Origin $+6 \Delta$ & 0.24 & 1.2 & Mc 3 & -0.24 & 5.6 & 16 & Mc 3 & 6.9 & $3,882,514.267$ \\
\hline 7 & Origin $+7 \Delta$ & 0.28 & 1.4 & Mc 3 & -0.28 & 5.7 & 17 & Mc 3 & 6.8 & $3,962,851.111$ \\
\hline 8 & Origin $+8 \Delta$ & 0.32 & 1.6 & Mc 3 & -0.32 & 5.8 & 18 & Mc 3 & 6.7 & $4,076,378.745$ \\
\hline
\end{tabular}

Simulations runs are made by simultaneously increasing (variables with positive step size) or decreasing (variables with negative step size) the value of the controlled factors. Table 5 shows the results from the Steepest Ascent Experiment when all three controllable factors are varied simultaneously.

Table 5: $\quad$ Steepest ascent experiment for the push system 


\begin{tabular}{|c|c|c|c|c|c|c|c|c|c|c|}
\hline 9 & Origin $+9 \Delta$ & 0.36 & 1.8 & Mc 3 & -0.36 & 5.9 & 19 & Mc 3 & 6.6 & $4,154,052.123$ \\
\hline 10 & Origin $+10 \Delta$ & 0.4 & 2 & Mc 3 & -0.4 & 6 & 20 & Mc 3 & 6.5 & $4,269,338.761$ \\
\hline 11 & Origin $+11 \Delta$ & 0.44 & 2.2 & Mc 3 & -0.44 & 6.1 & 21 & Mc 3 & 6.4 & $4,319,158.606$ \\
\hline 12 & Origin $+12 \Delta$ & 0.48 & 2.4 & Mc 3 & -0.48 & 6.2 & 22 & Mc 3 & 6.3 & $4,441,031.637$ \\
\hline 13 & Origin $+13 \Delta$ & 0.52 & 2.6 & Mc 3 & -0.52 & 6.3 & 23 & Mc 3 & 6.2 & $4,521,894.522$ \\
\hline 14 & Origin $+14 \Delta$ & 0.56 & 2.8 & Mc 3 & -0.56 & 6.4 & 24 & Mc 3 & 6.1 & $4,565,834.843$ \\
\hline 15 & Origin $+15 \Delta$ & 0.6 & 3 & Mc 3 & -0.6 & 6.5 & 25 & Mc 3 & 6 & $4,558,394.567$ \\
\hline 16 & Origin $+16 \Delta$ & 0.64 & 3.2 & Mc 3 & -0.64 & 6.6 & 26 & Mc 3 & 5.9 & $4,661,795.126$ \\
\hline 17 & Origin $+17 \Delta$ & 0.68 & 3.4 & Mc 3 & -0.68 & 6.7 & 27 & Mc 3 & 5.8 & $4,540,612.797$ \\
\hline 18 & Origin $+18 \Delta$ & 0.72 & 3.6 & Mc 3 & -0.72 & 6.8 & 28 & Mc 3 & 5.7 & $4,457,537.326$ \\
\hline 19 & Origin $+19 \Delta$ & 0.76 & 3.8 & Mc 3 & -0.76 & 6.9 & 29 & Mc 3 & 5.6 & $4,359,272.176$ \\
\hline 20 & Origin $+20 \Delta$ & 0.8 & 4 & Mc 3 & -0.8 & 7 & 30 & Mc 3 & 5.5 & $4,289,590.091$ \\
\hline 21 & Origin $+21 \Delta$ & 0.84 & 4.2 & Mc 3 & -0.84 & 7.1 & 31 & Mc 3 & 5.4 & $4,218,240.514$ \\
\hline 22 & Origin $+22 \Delta$ & 0.88 & 4.4 & Mc 3 & -0.88 & 7.2 & 32 & Mc 3 & 5.3 & $4,107,051.265$ \\
\hline 23 & Origin $+23 \Delta$ & 0.92 & 4.6 & Mc 3 & -0.92 & 7.3 & 33 & Mc 3 & 5.2 & $4,031,541.485$ \\
\hline 24 & Origin $+24 \Delta$ & 0.96 & 4.8 & Mc 3 & -0.96 & 7.4 & 34 & Mc 3 & 5.1 & $3,923,617.966$ \\
\hline 25 & Origin $+25 \Delta$ & 1 & 5 & Mc 3 & -1 & 7.5 & 35 & Mc 3 & 5 & $3,853,942.363$ \\
\hline
\end{tabular}

In Table 5, it can be seen that maximum profit is 4,661,795.126 Baht and it is received when the inter-arrival time is set at 6.6 minutes, buffer size is set at 26 units, bottleneck position is at machine 3 and the bottleneck processing time of 5.9 minutes. These values will be used further in phase II of the Response Surface Methodology. Fig. 4 below shows the trend of the steepest ascent experiment and plots the results received from Table 5.

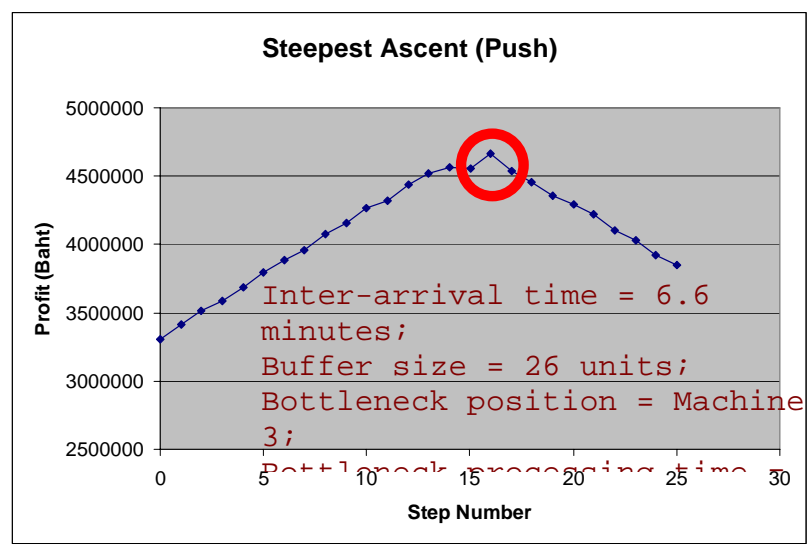

Fig. 4: $\quad$ Steepest ascent for the push system

Phase II: Second order analysis

The procedure of this phase is similar to the first phase of the first order model fitting. Here the central composite design is used for the second-order polynomial approximation. The optimum point received from the first order analysis is used as the starting point of the second order analysis. The factors studied for this stage are the same, inter-arrival time, buffer size and bottleneck processing time and like before, bottleneck position with remain fixed at machine 3 . This factorial design is composed of $2^{k}(k=3)$ factorial runs augmented with 6 axial runs $(2 k)$; $( \pm \alpha, 0,0),(0, \pm \alpha, 0)$ and $(0,0, \pm \alpha)$ and 4 center points. The value of $\alpha$ is defined as (number of treatments $)^{1 / 4}$, which is $\left(2^{3}\right)^{1 / 4}=1.682$. This gives $18(8+6+4)$ factorial runs which under 
four different noise settings will give 72 experimental conditions. Results from this factorial design are shown in Table 6.

Table 6: $\quad 2^{3}$ Factorial design for the push system

\begin{tabular}{|c|c|c|c|c|c|c|c|c|c|}
\hline \multirow{2}{*}{$\begin{array}{l}\text { Obser- } \\
\text { vation }\end{array}$} & \multicolumn{4}{|c|}{ Coded variables } & \multicolumn{4}{|c|}{ Natural variables } & \multirow[b]{2}{*}{ Profit (Baht) } \\
\hline & $\begin{array}{c}X_{I} \text { (Inter- } \\
\text { arrival) }\end{array}$ & $\begin{array}{c}X_{2} \text { (buffer } \\
\text { size) }\end{array}$ & $\begin{array}{c}X_{3} \\
\text { (position) }\end{array}$ & $\begin{array}{c}X_{4} \\
\text { (severity) }\end{array}$ & $\begin{array}{c}N T_{I} \\
\text { (minutes) }\end{array}$ & $\begin{array}{c}N T_{2} \\
\text { (units) }\end{array}$ & $\begin{array}{c}N T_{3} \\
\text { (machine) }\end{array}$ & $\begin{array}{c}N T_{4} \\
\text { (minutes) }\end{array}$ & \\
\hline 1 & -1 & -1 & Mc 3 & -1 & 6.5 & 25 & Mc 3 & 5.8 & $4,621,765.894$ \\
\hline 2 & -1 & -1 & Mc 3 & 1 & 6.5 & 25 & Mc 3 & 6 & $4,546,144.567$ \\
\hline 3 & -1 & 1 & Mc 3 & -1 & 6.5 & 27 & Mc 3 & 5.8 & $4,627,064.796$ \\
\hline 4 & -1 & 1 & Mc 3 & 1 & 6.5 & 27 & Mc 3 & 6 & $4,576,337.148$ \\
\hline 5 & 1 & -1 & Mc 3 & -1 & 6.7 & 25 & Mc 3 & 5.8 & $4,506,583.331$ \\
\hline 6 & 1 & -1 & Mc 3 & 1 & 6.7 & 25 & Mc 3 & 6 & $4,502,425.755$ \\
\hline 7 & 1 & 1 & Mc 3 & -1 & 6.7 & 27 & Mc 3 & 5.8 & $4,540,612.797$ \\
\hline 8 & 1 & 1 & Mc 3 & 1 & 6.7 & 27 & Mc 3 & 6 & $4,479,351.965$ \\
\hline 9 & -1.682 & 0 & Mc 3 & 0 & 6.4 & 26 & Mc 3 & 5.9 & $4,673,951.334$ \\
\hline 10 & 1.682 & 0 & Mc 3 & 0 & 6.8 & 26 & Mc 3 & 5.9 & $4,451,360.618$ \\
\hline 11 & 0 & -1.682 & Mc 3 & 0 & 6.6 & 24 & Mc 3 & 5.9 & $4,565,834.403$ \\
\hline 12 & 0 & 1.682 & Mc 3 & 0 & 6.6 & 28 & Mc 3 & 5.9 & $4,564,651.324$ \\
\hline 13 & 0 & 0 & Mc 3 & -1.682 & 6.6 & 26 & Mc 3 & 5.7 & $4,567,420.318$ \\
\hline 14 & 0 & 0 & Mc 3 & 1.682 & 6.6 & 26 & Mc 3 & 6.1 & $4,508,364.513$ \\
\hline 15 & 0 & 0 & Mc 3 & 0 & 6.6 & 26 & Mc 3 & 5.9 & $4,661,795.126$ \\
\hline 16 & 0 & 0 & Mc 3 & 0 & 6.6 & 26 & Mc 3 & 5.9 & $4,614,114.837$ \\
\hline 17 & 0 & 0 & Mc 3 & 0 & 6.6 & 26 & Mc 3 & 5.9 & $4,622,017.674$ \\
\hline 18 & 0 & 0 & Mc 3 & 0 & 6.6 & 26 & Mc 3 & 5.9 & $4,629,352.835$ \\
\hline
\end{tabular}

Second order regression line is fitted to the data of this phase and the equation of the best fit line is:

$$
\begin{gathered}
y=4,631,849-52,476.4 X_{I}+3,254.972 X_{2}-21,313.1 X_{4} \\
-24,577.9 X_{I}^{2}-23,663.5 X_{2}^{2}-33,331 X_{4}^{2}
\end{gathered}
$$

Analysis of Variance is also carried out to check the adequacy of the second order model. The second-order equation gives F-value of 3.941 ( $p$-value of 0.001 ), which indicates that the model is adequate under $95 \%$ confidence level.

\section{Phase III: Optimum solution}

To find the optimum values of controlled factors that maximize the response, partial derivatives of all variables are taken and set to 0 . They are:

$$
\begin{gathered}
\frac{\partial Y}{\partial X_{1}}=-52,476.4-49,155.8 X_{1}=0 \\
\frac{\partial Y}{\partial X_{2}}=3,254.972-47,327 X_{2}=0
\end{gathered}
$$




$$
\frac{\partial Y}{\partial X_{4}}=-21,313.1-66,662 X_{4}=0
$$

After solving the equations, the level of controlled variables that generate the near optimal solution are at inter-arrival time $=6.493$ minutes, buffer size $=26.069$ units, bottleneck position $=$ machine 3 and bottleneck processing time $=5.868$ minutes. The buffer size is rounded to the nearby unit, giving us 26 units and the bottleneck processing time is rounded up to 5.9 minutes. This combination generates a profit of $4,659,859.51$ Baht when the values of independent variable are substituted into the regression equation.

Phase IV: Result verification

We have also managed to put the recommended parameter setting above into our simulation model and checked the profit generated from the model. It gives the profit of 4,675,869.01 Baht. This value is very close to the value received from the equation with the percentage difference of only $0.34 \%$. As a result, it can be concluded that the obtained results from the integrated approach are reliable and not arbitrary. Table 7 summarizes the optimum set of parameters received for the push driven flow line.

Table 7: $\quad$ Optimal solution for the push system

\begin{tabular}{ll}
\hline Part inter-arrival time & 6.493 minutes \\
\hline Buffer size & 26 units \\
Bottleneck position & At machine 3 \\
Bottleneck processing time & 5.9 minutes \\
\hline
\end{tabular}

\section{PULL SYSTEM}

In the interests of brevity, we will comment only on selected results since most of the processes are similar manner to the analysis of the push system.

\subsection{Taguchi method for experimental design}

The results where the $S / N$ ratios are highest in each controllable factor have been highlighted and shown in Table 8.

Table 8: $\quad$ S/N Ratios for all controlled factors of the pull system

\begin{tabular}{lccc}
\hline \multirow{2}{*}{ Controllable factors } & Low & Medium & High \\
\cline { 2 - 4 } & 130.454 & 128.390 & 122.713 \\
\hline Inter-arrival time & 126.749 & 127.384 & 127.423 \\
Number of kanbans & 127.229 & 127.117 & 127.211 \\
Bottleneck position & 126.688 & 127.445 & 127.424 \\
Bottleneck processing time & & & \\
\hline
\end{tabular}


The most robust design as recommended by the Taguchi method is the line designed with the inter-arrival time of 5 minutes, 15 kanbans, bottleneck position at machine 1 and bottleneck processing time of 7.5 minutes.

\subsection{Response Surface Methodology}

Phase I: First order analysis

\section{Step 1: Range determination}

The region of exploration is set as following: $(2.5,7.5)$ for inter-arrival time (minutes) where the center point is 5 minutes, $(10,20)$ for number of kanbans where the center point is 15 kanbans, bottleneck position at machine 1 and finally $(5,10)$ minutes for bottleneck processing time where the center point is at 7.5 minutes.

\section{Step 2: Coding independent variables}

The coded variables are: $X_{1}=($ inter-arrival time -5$) / 2.5 ; X_{2}=($ kanban -15$) / 5 ; X_{4}=$ (bottleneck processing time - 7.5) / 2.5 where $X_{1}, X_{2}, X_{4}$ are coded variables of part inter-arrival time, number of kanbans and bottleneck processing time respectively.

\section{Step 3: Data collection}

$2^{k}(k=3)$ full factorial design is used and augmented by four center points. As a result, there are 48 experimental conditions in all.

\section{Step 4: First order model fitting}

The data collected in Step 3 is used and a first order model that best fits the data is found. Here $X_{1}, X_{2}$, and $X_{4}$ are the independent variables (controlled factors) and $y$ is the profit. The regression equation is as follows:

$$
y=3,200,960+322,066.9 X_{1}+67,778.248 X_{2}-938,672 X_{4}
$$

Step 5: First order adequacy test

The first-order equation gives F-value of 341.652 (p-value of 0.000 ), which indicates that the model is adequate.

\section{Step 6: Method of steepest ascent}

The largest regression coefficient in the model comes from $X_{4}$ (bottleneck processing time) with $\beta_{4}$ of 938,672 . The coded step size for other variables can be calculated by the following equation:

$$
\Delta X_{i}=\beta_{i} / \beta_{4} \quad \text { for } \quad i=1,2,4 .
$$

Hence,

$$
\begin{aligned}
& \Delta X_{1}=322,066.9 / 938,672=0.343 \\
& \Delta X_{2}=67,778.248 / 938,672=0.072 \\
& \Delta X_{4}=-938,672 / 938,672=-1 .
\end{aligned}
$$


Next the coded variable $\Delta X_{i}$ is converted to natural variable, $N T_{\mathrm{i}}$. This is done by multiplying $\Delta X_{i}$ with the actual step size $\left(S_{i}\right)$. The smallest step size for the inter-arrival time is set at 0.1 minutes, for the number of kanbans is set at 1 and for the bottleneck processing time is set at 0.1 minutes.

Therefore,

$$
\begin{aligned}
& \triangle X_{I} S_{l}=0.1 \text { minute } \quad \text { So, } S_{l}=0.1 / 0.343=0.27 \\
& \Delta X_{2} S_{2}=1 \text { kanban } \quad \text { So, } S_{2}=1 / 0.072=13.89 \\
& \Delta X_{4} S_{4}=0.1 \text { minute So, } S_{4}=-0.1 /-1=0.1 \text {. }
\end{aligned}
$$

Figure 5 shows the results from the Steepest Ascent Experiment when all three controllable factors are varied simultaneously. It can be seen that maximum profit is 4,786,334.323 Baht and it is received when the inter-arrival time is set at 6.4 minutes, number of kanbans equal to 29, bottleneck position is fixed at machine 1 and bottleneck processing time of 6.1 minutes. These values will be used further in phase II of the Response Surface Methodology.

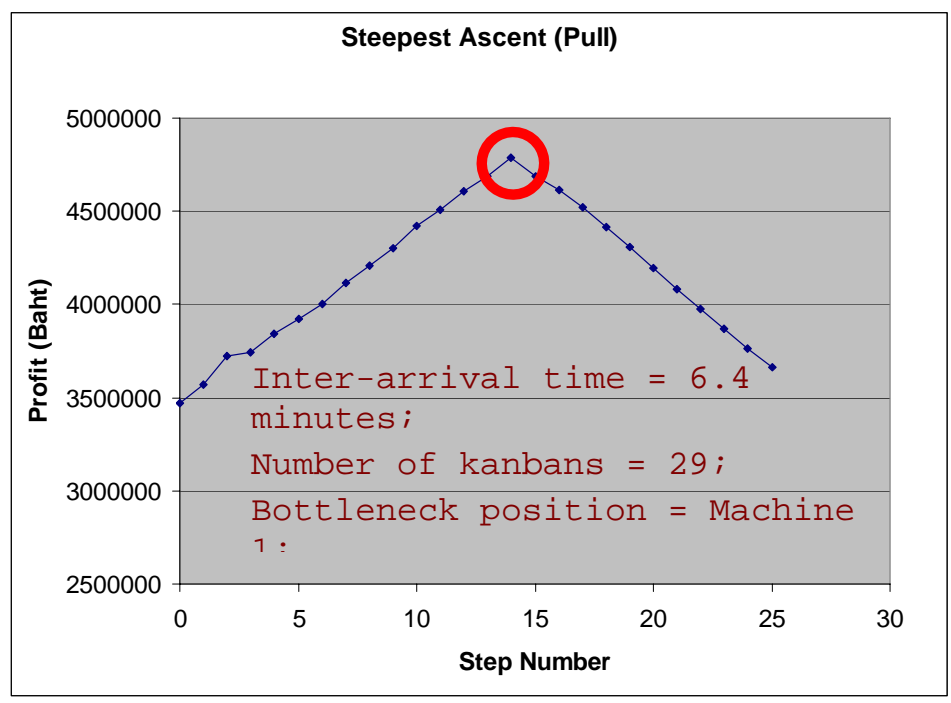

Fig. 5: $\quad$ Steepest ascent for the pull system

Phase II: Second order analysis

Second order regression is fitted to the data obtained from $2^{3}$ factorial runs augmented with 6 axial runs and 4 center points around the optimal point received from the first order analysis. The equation of the best fit line is:

$$
\begin{gathered}
y=4,771,553-11,724.8 X_{1}+32,803.039 X_{2}-6,354.048 X_{4} \\
-34,232.2 X_{1}^{2}-21,352.1 X_{2}^{2}-32,809.5 X_{4}^{2}
\end{gathered}
$$

Analysis of Variance is carried out to check the adequacy of the second order model. The second-order equation gives F-value of 3.102 (p-value of 0.005), which indicates that the model is adequate under $95 \%$ confidence level. 
Phase III: Optimum solution

To find the optimum values of controlled factors that maximize the response, partial derivatives of all variables are taken and set to 0 . They are:

$$
\begin{aligned}
& \frac{\partial Y}{\partial X_{1}}=-11,724.8-68,464.4 X_{1}=0 \\
& \frac{\partial Y}{\partial X_{2}}=32,803.039-42,704.2 X_{2}=0 \\
& \frac{\partial Y}{\partial X_{4}}=-6,354.048-65,619 X_{4}=0
\end{aligned}
$$

After solving the equations, the level of controlled variables that generate the near optimal solution are at inter-arrival time $=6.383$ minutes, number of kanbans $=29.078$, bottleneck position $=$ machine 1 and bottleneck processing time $=6.09$ minutes. The number of kanbans is rounded to the nearby unit, giving us 29 kanbans and the bottleneck processing time is rounded up to 6.1 minutes. This combination generates a profit of 4,772,556.91 Baht when the values of independent variable are substituted into the regression equation.

\section{Phase IV: Result verification}

The verified simulation run gives the profit of $4,801,336.42$ Baht. This value is very close to the value we receive from the equation with the percentage difference of only $0.6 \%$. Table 9 summarizes the optimum set of parameters received for the pull driven flow line.

Table 9: $\quad$ Optimal solution for the pull system

\begin{tabular}{ll}
\hline Part inter-arrival time & 6.383 minutes \\
\hline Number of kanbans & 29 kanbans \\
Bottleneck position & At machine 1 \\
Bottleneck processing time & 6.1 minutes \\
\hline
\end{tabular}

\section{COMPARISON BETWEEN THE PUSH AND PULL SYSTEMS}

The main purpose of this study is to study the impact of push and pull mechanisms under optimal settings on the economic consideration of the production line with a constraint resource. At the optimal design, it is found that the pull system can generate a higher profit (about $2.7 \%$ higher). The best location of the bottleneck of the push system is at the middle station in the line (machine 3) where it is at the first station (machine 1) for the pull system. This outcome reflects the economic consideration's view point. For the push system where the customer order enters from the first machine, it prefers to limit the number of arrival parts into the system by having the bottleneck located earlier in the line. If the bottleneck is located later in the line, higher inventory would be built up in front of the bottleneck machine and this may cause too expensive inventory holding cost and possible process blocking. From the experiment, there is quite close $S / N$ ratio given from the Taguchi method between placing the bottleneck at machine 1 and machine 3 (see Fig. 3) but the $S / N$ ratio of the bottleneck position at machine 3 is slightly higher. This results from trading off between the higher lost sales cost and the lower inventory 
holding cost when the bottleneck is located at the first machine. With our experiment setting and cost structure, placing the bottleneck at the machine 3 is shown to have slightly higher $S / N$ ratio.

For the pull system, the number of inventory in the line has already been limited by the number of kanbans no matter where the bottleneck is located. As a result, the pull system where the customer order is used to pull parts out from the end of the line (last machine) would prefer to have the bottleneck placing further away from the last machine. In this way, the bottleneck would not block the incoming orders and this results in lower number of overflow orders and cheaper lost sales cost. In order to investigate the impact of the push and pull mechanisms on the system under each cost category, total costs need to be broken down into each individual cost as presented in Table 10 .

Table 10: $\quad$ Cost break-down of the push and pull systems

\begin{tabular}{lcc}
\hline \multicolumn{1}{c}{ Activity costs } & Push system (Baht)* & Pull system (Baht)* \\
\hline Machine operating cost & 296,486 & 298,231 \\
Raw material cost & $869,818.8$ & $878,266.3$ \\
Machine repairing cost & $96,046.2$ & $96,920.3$ \\
Holding cost & $35,983.79$ & $106,762.1$ \\
Machine idle cost & $133,903.1$ & $129,768.5$ \\
Lost sales cost & $26,303.75$ & 27,400 \\
Late penalty cost & $88,057.04$ & 447.56 \\
Bottleneck time reduction cost & 738,000 & 702,000 \\
\hline$*$ Remark: all costs are averaged over 4 noise settings for one replication length (115,200 minutes) \\
\hline \multicolumn{2}{c}{ Push system (minutes) } & Pull system (minutes) \\
\hline Customer lead time & 279.585 & 70.532 \\
\hline \# time from an order arrival till the time that the order has been finished & \\
\hline
\end{tabular}

From Table 10, the push system shows lower costs from the machine operating, raw material, and inventory holding. The machine operating cost and raw material cost of the pull system are more expensive due to the fact that the pull system at its optimal design operates with a slightly higher demand level (the pull system's part inter-arrival time of 6.383 minutes as compared to 6.493 minutes of the push system). This definitely causes higher requirement of raw materials and longer machine operation time. In addition, as the pull system needs to hold up to 29 kanbans between machines, higher holding cost needs to be paid for this amount of inventory whether or not there is a customer demand.

However, the pull system can operate cheaper from the machine idle cost, bottleneck time reduction cost and late penalty cost. The cheaper machine idle cost of the pull system can be explained by a higher number of units produced as discussed previously. The operating time at the bottleneck of the pull system is reduced from the normal level of 10 minutes to 6.1 minutes as compared to 5.9 minutes of the push system. As a result, the push system needs to pay higher processing time reduction cost. Regarding the cheaper late penalty cost of the pull system, since the pull system can operate with a much shorter lead time, a lower late penalty cost is charged as a result. By keeping a number of kanbans between succeeding stations, the pull system is shown to respond to the demand more quickly and provides much shorter customer lead time. However, it has to pay the price by having a higher inventory holding cost. 


\section{CONCLUSION}

Level of demand, buffer size (number of kanbans), bottleneck position and level of bottleneck are given consideration. Using the integrated approach of Taguchi method along with Response Surface Methodology has enabled us to find the optimal setting of the flow line under push and pull driven mechanisms where qualitative and quantitative factors are present simultaneously. From the study, we have shown that this approach can be used to maximize the profit generated from the line under these different production control systems. Taguchi method provides the most robust design in the range of parameters considered and the response surface methodology further improves these results to find the optimal designs of the studied flow line.

Finally, despite the benefits of the integrated approach, there are also some drawbacks that need to be kept in mind. As poor inputs will lead to poor results, the range of each parameter setting and accuracy of the employed cost structure will play a major role in obtaining good results. Although this approach attempts to optimize manufacturing system design and determine a region in the factor space where operating specifications are met, the generated set of optimal parameters may fall into a local optimum only. However, in the case of a well planned experiment with a well defined factor space, the true optimum can be achieved.

\section{REFERENCES}

1. Sarker, B.R. and Fitzsimmons, J.A. (1989), The Performance of Push and Pull Systems: A Simulation and Comparative Study, International Journal of Production Research, vol. 26, no. 10, pp. 1715-1731.

2. Lee, L.C. (1988), A Comparative Study of the Push and Pull Production Systems, International Journal of Production Management, vol. 9, no. 4, pp. 5-18.

3. Browne, J., Harden, J., and Shivnan, J. (1988), Production Management Systems, Addison-Wesley, England.

4. Pegden, C.D., Shannon, R.E., and Sadowski, R.P. (1995), Introduction to Simulation Using Siman, Second Edition, McGraw-Hill, Inc, Singapore.

5. Dallery, Y. and Gershwin, S.B. (1992), Manufacturing Flow line Systems: A Review of Models and Analytical Results, Queueing Systems, vol. 12, pp. 3-94.

6. Blackstone, J.H., Phillips, D.T., and Hogg, G.L. (1982), A State-of-the-art Survey of Dispatching Rules for Manufacturing Job Shop Operations, International Journal of Production Research, vol. 20, pp. 27-45.

7. Powell, S.G. and Pyke, D.F. (1996), Allocation of Buffers to Serial Production Lines with Bottlenecks, IIE Transactions, vol. 28, pp. 18-29.

8. Buzacott, J.A. and Shantikumar, J.G. (1993), Stochastic Models of Manufacturing Systems, Prentice Hall, New York.

9. Antony, J. (20`01), Simultaneous optimization of multiple quality characteristics in manufacturing process using Taguchi's quality loss function, International Journal of Advanced Manufacturing Technologies, vol. 17, pp. 134-138.

10. Montgomery, D.C. (1991), Design and Analysis of Experiments, $3^{\text {rd }}$ Edition, John Wiley \& Sons, Singapore.

11. Shang, J.S. (1995), Robust design and optimization of material handling in an FMS, International Journal of Production Research, vol. 33, no. 9, pp. 2437-2454.

12. Shang, J.S. and Tadikamalla, P.R. (1998), Multicriteria design and control of a cellular manufacturing system through simulation and optimization, International Journal of Production Research, vol. 36, no. 6, pp. 1515-1528. 